\section{BMJ Open} Ophthalmology

\title{
Diabetic maculopathy: multicolour and SD-OCT versus fundus photography
}

\author{
Obaid Kousha (1) , ${ }^{1}$ Martina Maria Delle Fave, ${ }^{2}$ Mariano Cozzi, ${ }^{3}$ Elisa Carini, ${ }^{4}$ \\ Sergio Pagliarini ${ }^{5}$
}

To cite: Kousha 0 ,

Delle Fave MM, Cozzi M, et al. Diabetic maculopathy: multicolour and SD-OCT versus fundus photography. BMJ Open Ophthalmology

2021;6:e000514. doi:10.1136/ bmjophth-2020-000514

Received 19 May 2020 Revised 8 November 2020 Accepted 8 February 2021
Check for updates

C) Author(s) (or their employer(s)) 2021. Re-use permitted under CC BY-NC. No commercial re-use. See rights and permissions. Published by BMJ.

${ }^{1}$ Department of Ophthalmology, Ninewells Hospital, Dundee, UK ${ }^{2}$ Department of Ophthalmology, Free University of Brussels,

Brussels, Belgium

${ }^{3}$ Department of Biomedical and Clinical Science, Luigi Sacco University Hospital, Milano, Lombardia, Italy

${ }^{4}$ Eye Clinic, Department of Biomedical and Clinical Science, Luigi Sacco University Hospital, Milano, Lombardia, Italy ${ }^{5}$ Department of Ophthalmology, University Hospitals Coventry and Warwickshire NHS Trust, Coventry, UK

Correspondence to Dr Obaid Kousha; okousha@ gmail.com

\section{ABSTRACT}

Objective The English Diabetic Eye Screening (DES) programme recommends patients with M1 diabetic maculopathy to be referred to hospital eye services. DES uses flash fundus photography as the reference standard for maculopathy grading. We compared multicolour versus non-stereoscopic fundus photography at identifying $\mathrm{M} 1$ maculopathy, with spectral domain optical coherence tomography (SD-OCT) identifying macular thickening. Methods and analysis This cross-sectional study included 345 patients with R1M1 referred from DES and reviewed in secondary care with fundus photographs, multicolour and SD-OCT. Maculopathy was graded based on DES exudate criteria on both multicolour and fundus photography in a blind fashion by two independent graders. Macular thickness was ascertained on SD-OCT. Results Intergrader agreement on grading maculopathy using fundus photography (Cohen's $\kappa=0.91$ ) and multicolour (Cohen's $\kappa=0.82$ ) was 'almost perfect'. Agreement between fundus photography and multicolour on grading maculopathy (Cohen's $\kappa=0.76$ ) was 'substantial'. Compared with fundus photography, multicolour had sensitivity of $87 \%(95 \% \mathrm{Cl} 81 \%$ to $93 \%)$ and specificity of $90 \%(95 \% \mathrm{Cl} 87 \%$ to $94 \%)$ in detecting M1 maculopathy. SD-OCT identified 84 eyes with macular thickening, 47 of which were graded as MO by fundus photography. 5 eyes with exudates and severe macular oedema requiring urgent intervention were also missed on fundus photography but not on multicolour. Multicolour, when complemented by SD-OCT, did not miss any clinically significant macular oedema.

Conclusion Multicolour integrates synergistically in a single platform with SD-OCT providing effective monitoring of M1 diabetic maculopathy. The need for fundus photography is eliminated by multicolour/SD-OCT in dedicated R1M1 virtual clinics not requiring parallel diabetic retinopathy grading.

\section{INTRODUCTION}

In the UK, up to one-third of adults with diabetes have concurrent diabetic eye disease. ${ }^{12}$ It is the most common cause of sight loss among working age adults. ${ }^{3}$ Therefore, the English National Health Service Diabetic Eye Screening (DES) programme offers diabetic eye screening for individuals with diabetes aged 12 years and over. ${ }^{3}$

Diabetic maculopathy manifesting as diabetic macular oedema (DMO) is the

\section{Key messages}

What is already known about this subject?

- Diabetic retinopathy is the most common cause of sight loss among working age adults. The English NHS Diabetic Eye Screening (DES) programme recommends patients with M1 diabetic maculopathy, diagnosed using flash fundus photography, to be referred to hospital eye services.

\section{What are the new findings?}

This is a cross-sectional study comparing fundus photography (FP) with MultiColor (MC) integrated with SD-OCT in diagnosing M1 maculopathy. As compared to FP, MC has excellent sensitivity and specificity in diagnosing M1 maculopathy. Furthermore, MC integrates synergistically in a single platform with SD-OCT providing effective monitoring of M1 diabetic maculopathy, identifying potential patients requiring treatment with the modern pharmacologic agents such as vascular endothelial growth factor inhibitors (anti-VEGF), eliminating the need for FP in dedicated R1M1 virtual clinics not requiring paralle diabetic retinopathy grading.

How might these results change the focus of research or clinical practice?

- The DES criteria for maculopathy grading using nonstereoscopic FP is becoming increasingly less relevant in an era of anti-VEGF where OCT is becoming more important in diagnosing diabetic maculopathy. This study does not provide a direct evaluation of MC imaging and FP within the DES programme.

leading cause of blindness among the population with diabetes. ${ }^{4}$ The main pathophysiological factor is thought to be the disruption of blood-retina barrier secondary to long-term hyperglycaemic insult and inflammation, which leads to leakage of plasma into the neurosensory retina resulting in DMO and hard exudates in the macula. ${ }^{45}$ The hard exudates are composed of lipids and plasma proteins accumulating mainly at the outer plexiform layer. ${ }^{6-8}$

The DES defines the macula as the area of the retina confined by a circle with the fovea at its centre and touching the temporal edge of the optic disc. ${ }^{9}$ The DES recommends 
patients with M1 diabetic maculopathy to be referred to the hospital eye services (HES). ${ }^{3}$ The DES definition of M1 maculopathy includes any exudates within one disc diameter (1DD) of the fovea, retinal thickening within 1DD of the fovea or a group of exudates that covers an area that is greater than or equal to half the disc area, all within the macula. ${ }^{910}$

The DES uses flash colour and red-free fundus photography (FP) as the reference standard for maculopathy grading. ${ }^{11}$ Colour FP is an imaging modality which closely concords with clinical examination findings when using an ophthalmoscope or slit-lamp biomicroscope. However, in the past decade, different systems based on confocal scanning laser ophthalmoscopy (SLO) for fundus imaging have been developed producing 'pseudo-colour' composites. Examples of these systems include Optos wide-field SLO (Optos, Dunfermline, UK) using green $(\lambda=532 \mathrm{~nm})$ and red $(\lambda=633 \mathrm{~nm})$ laser wavelengths ${ }^{12}$; Nidek F-10 confocal SLO (Nidek, Fremont, California, USA) which uses infrared $(\lambda=790 \mathrm{~nm})$, red $(\lambda=660 \mathrm{~nm})$, green $(\lambda=532 \mathrm{~nm})$ and blue $(\lambda=490$ $\mathrm{nm})$ laser wavelengths ${ }^{13}$; multicolour (MC) confocal SLO imaging (Heidelberg Engineering, Heidelberg, Germany) which uses three simultaneously acquired laser reflectance images using infrared $(\lambda=815 \mathrm{~nm})$, green $(\lambda=518 \mathrm{~nm})$ and blue $(\lambda=486 \mathrm{~nm})$ wavelengths. ${ }^{14}$ Fundus images obtained using systems such as MC can appear strikingly different when compared with the FP, however, some macular pathologies appear more distinct on the MC. ${ }^{1415}$

The additional benefit of MC system is that it is integrated with spectral domain optical coherence tomography (SDOCT) in a single platform. This potentially allows for a more comprehensive evaluation of diabetic maculopathy, based on detection of exudates and retinal thickening, when compared with non-stereoscopic FP alone. Indeed, there is a good correlation between macular fluorescein angiographic leakage and visual acuity with OCT features of diabetic maculopathy. ${ }^{16} 17$

In this study, first, we aim to compare MC versus FP at identifying M1 maculopathy based on presence of macular exudates. Second, we endeavour to identify M1 maculopathy based on retinal thickening with SD-OCT, which is undetectable on non-stereoscopic FP. Finally, we compare the merits of integration of MC and SD-OCT versus $\mathrm{FP}$ when evaluating M1 maculopathy in dedicated R1M1 virtual clinics not requiring parallel diabetic retinopathy grading.

\section{METHOD}

\section{Population and image acquisition}

This is a cross-sectional study of 187 patients with diabetes (367 eyes) who were monitored in virtual macular clinics between 1 January 2012 and 1 January 2013 after an initial referral from the English DES programme to HES for further evaluation of R1M1 grading (figure 1). Mydriasis was achieved $30 \mathrm{~min}$ after instillation of preservative-free tropicamide $1 \%$ and phenylephrine $2.5 \%$ eye drops. A

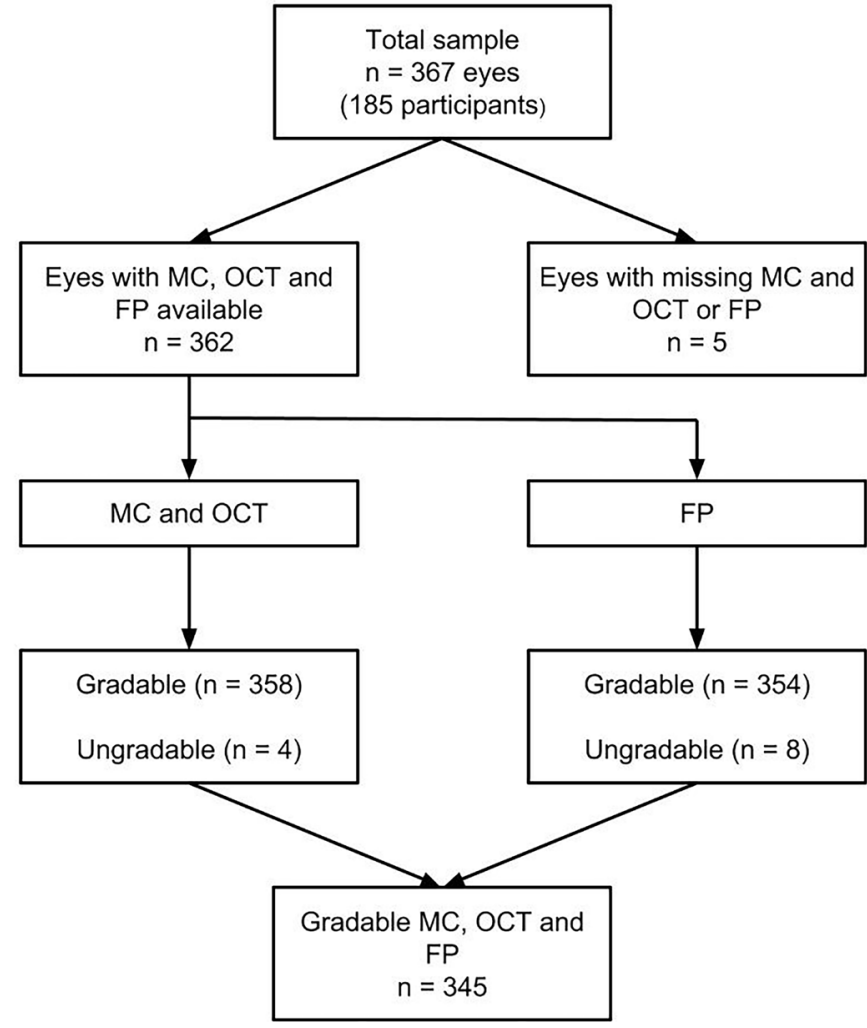

Figure 1 Flowchart of study participants. FP, fundus photography; MC, multicolour; OCT, optical coherence tomography.

Zeiss Visucam Pro NM non-mydriatic fundus camera was used to obtain FPs consisting of $30^{\circ}$ and $45^{\circ}$ colour and red-free images centred on the fovea. Heidelberg Spectralis (Heidelberg Engineering, Heidelberg, Germany) was used to acquire $30^{\circ} \mathrm{MC}$ images centred on the fovea as well as SD-OCT scans. Specifically, we used the built-in Fast Macula protocol of $20^{\circ} \times 15^{\circ} 6 \times 6 \mathrm{~mm}$ volume centred on the fovea, consisting of 19 lines spaced $240 \mu \mathrm{m}$ of 512 A-Scans each.

\section{Retinal grading}

\section{Exudate criteria}

FP images were viewed using Zeiss VISUPAC software with its available image manipulation tools applied if needed. MC (including the individual blue, green and infrared reflectance images) and SD-OCT images were viewed using Heidelberg Eye Explorer with its image manipulation tools used as necessary.

The MC and FP images were delinked. The diabetic maculopathy was graded based on exudate criteria as described by the DES on both MC and FP in a blind fashion independently by two graders (OK and SP). Infrared reflectance, green reflectance, blue reflectance and $\mathrm{MC}$ composite were assessed individually to decide on the presence of exudates (figure 2). An image was deemed ungradable if the detail of the third order retinal vessels from the superior and inferior vascular arcades was not discernible. 


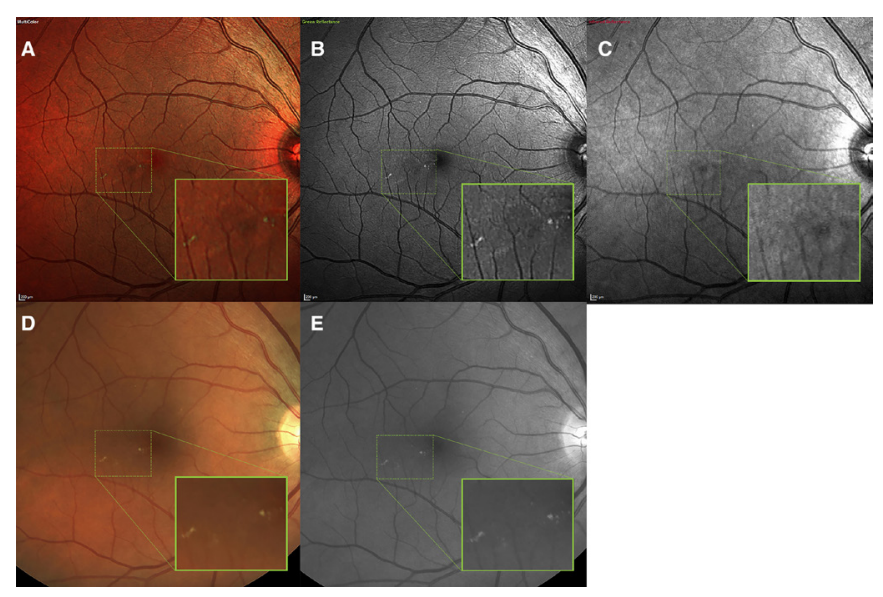

Figure 2 Visualisation of exudates on different imaging modalities. Macular exudates on multicolour composite (A), green reflectance channel $(B)$, infrared reflectance channel (C), fundus photography (FP) (D) and red-free FP (E). The green reflectance channel is the main contributor to exudate visualisation on the multicolour composite image. Please note the absence of exudate on infrared reflectance channel as it images deeper retinal structures.

On SD-OCT, an exudate was defined as a homogenous hyper-reflective focus of $\geq 63 \mu \mathrm{m}$ at, or internal to the outer plexiform layer with no inner hyporeflectivity. M1 maculopathy based on exudate criteria as outlined by the DES was not assessed systematically using SD-OCT as the spacing between the B-scans was $>63 \mu \mathrm{m}$ and the scan did not cover the entire macular as defined by the DES. However, any exudate seen on SD-OCT was recorded and correlated with FP and MC findings.

\section{Macular thickness criteria}

Macular oedema and thickness were ascertained on SD-OCT and retinal thickness maps on Heidelberg Eye Explorer by two independent graders ( $\mathrm{OK}$ and SP). The thickness map was centred at the fovea and the retinal boundaries were defined between the internal limiting membrane and the Bruch's membrane/retinal pigment epithelium complex-correction of the automated segmentation was made if needed. We defined abnormally increased macular thickening as central subfield thickness (CST) of $\geq 320 \mu \mathrm{m}$ for men and $\geq 310$ $\mu \mathrm{m}$ for women or thickening of $\geq 360 \mu \mathrm{m}$ in any of the Early Treatment Diabetic Retinopathy Study (ETDRS) grid inner sectors on Heidelberg Spectralis SD-OCT as described elsewhere. ${ }^{18} 19$ 'Clinically significant macular oedema' (CSMO) on SD-OCT was defined as spongiform or multicystoid retinal thickening of $\geq 370 \mu \mathrm{m}$ in the CST or of $>390 \mu \mathrm{m}$ in any of the ETDRS grid inner sectors. ${ }^{19}$ A correction factor of $+70 \mu \mathrm{m}$ was used to convert Stratus OCT (Carl Zeiss, Oberkochen, Germany) reported values for retinal thickening in the literature to equivalent Heidelberg Spectralis SD-OCT. ${ }^{20}$ Eyes with other causes of increased macular thickness such as vitreomacular traction, epiretinal membrane and choroidal neovascularisation were excluded. Any disagreements between the two graders (OK and SP) were resolved by a third grader (MMDF).

\section{Patient and public involvement}

Patients were not directly involved in the design of this study.

\section{Statistical analysis}

Statistical analysis was performed using IBM SPSS Statistics for Windows, V.22. The tests were two tailed with type I error set at $\alpha=0.05$. To ascertain the level of agreement between the two graders (OK vs SP) and the two different imaging modalities (MC vs FP), Cohen's $\kappa$ coefficient was calculated. ${ }^{21} \mathrm{FP}$ was set as the reference standard and the sensitivity, the specificity, the positive predictive value and the negative predictive value of $\mathrm{MC}$ at detecting $\mathrm{M} 1$ maculopathy as per DES were calculated with $95 \%$ CIs.

\section{RESULT}

The study included 345 eyes with gradable images in all three modalities (figure 1). Either FP or MC image was missing from five eyes. There were four ungradable MC images and eight ungradable FP images. The rate of M1 diabetic maculopathy in this cohort was $32 \%$, and $35 \%$ on FP and MC, respectively (table 1).

Intergrader and intermodality level of agreement in detecting M1 diabetic maculopathy based on exudate criteria of DES

The levels of agreements are listed in table 2. There was an 'almost perfect' intergrader (OK vs SP) level of agreement in detecting M1 maculopathy on FP (Cohen's $\kappa=0.91 ; \mathrm{p}<0.0001)$. There was a 'substantial' intergrader (OK vs SP) level of agreement in detecting M1 maculopathy on MC (Cohen's $\kappa=0.82$; $\mathrm{p}<0.0001$ ). The intermodality level of agreement between MC and FP in detecting M1 maculopathy was also 'substantial' (Cohen's $\kappa=0.76 ; \mathrm{p}<0.0001$ for $\mathrm{OK}$ and Cohen's $\kappa=0.72$; $\mathrm{p}<0.0001$ for SP). Furthermore, once the final grading for each patient was agreed on FP and MC, the intermodality level of agreement remained 'substantial' (Cohen's $\kappa=0.76 ; \mathrm{p}<0.0001)$.

The diagnostic performance of MC in detecting M1 diabetic maculopathy based on exudate criteria of DES compared with FP as reference standard

Compared with the FP reference standard, the MC had sensitivity of $87 \%$ (95\% CI $81 \%$ to $93 \%$ ), specificity of $90 \%$ ( $95 \%$ CI $87 \%$ to $94 \%$ ), positive predictive value of $80 \%$ (95\% CI $73 \%$ to $88 \%$ ) and negative predictive value of $94 \%$ (95\% CI $91 \%$ to $97 \%$ ) in detecting M1 maculopathy. In the peripheral macula, beyond 1DD of the fovea, where M1 is defined by group of exudates rather than any exudates, MC had sensitivity of $97 \%$ (95\% CI 92\% to $100 \%$ ), specificity of $99 \%$ (95\% CI $98 \%$ to $100 \%$ ), positive predictive value of $95 \%$ (95\% CI $87 \%$ to $100 \%$ ) and negative predictive value of $99 \%$ (95\% CI $99 \%$ to $100 \%$ ) for detection of M1 maculopathy. 


\begin{tabular}{|c|c|}
\hline \multicolumn{2}{|l|}{ M1 diabetic maculopathy (DES exudate criteria) } \\
\hline On FP, n (\%) & $109(32)$ \\
\hline On MC, n (\%) & $119(35)$ \\
\hline \multicolumn{2}{|c|}{$\begin{array}{l}\text { M1 diabetic maculopathy (DES exudate criteria) without } \\
\text { macular thickening on SD-OCT }\end{array}$} \\
\hline FP & 76 \\
\hline MC & 80 \\
\hline \multicolumn{2}{|l|}{ Macular thickening on SD-OCT } \\
\hline $\begin{array}{l}\text { Thickened CST (male } \geq 320 \mu \mathrm{m} \text { and female } \\
\geq 305 \mu \mathrm{m} \text { ) }\end{array}$ & 40 \\
\hline $\begin{array}{l}\text { Thickened inner sector of ETDRS grid } \\
(\geq 360 \mu \mathrm{m})\end{array}$ & 70 \\
\hline Thickened CST and/or inner sector & 84 \\
\hline $\begin{array}{l}\text { Thickened CST and/or inner sector but no } \\
\text { exudates on FP }\end{array}$ & 47 \\
\hline $\begin{array}{l}\text { Thickened CST and/or inner sector but no } \\
\text { exudates on MC }\end{array}$ & 42 \\
\hline $\begin{array}{l}\text { Thickened CST and/or inner sector but no } \\
\text { exudates on FP or MC }\end{array}$ & 37 \\
\hline \multicolumn{2}{|l|}{ 'Clinically significant macular oedema' } \\
\hline Total & 18 \\
\hline Missed by MC & 1 \\
\hline Missed by FP & 5 \\
\hline \multicolumn{2}{|l|}{ Other macular abnormalities } \\
\hline Vitreomacular traction & 6 \\
\hline Subfoveal pigment epithelial detachment & 1 \\
\hline Epiretinal membrane & 4 \\
\hline Central serous chorioretinopathy & 1 \\
\hline
\end{tabular}

CST, central subfield thickness; DES, Diabetic Eye Screening; ETDRS, Early Treatment Diabetic Retinopathy Study; FP, fundus photography; MC, multicolour; SD-OCT, spectral domain optical coherence tomography.

\section{Macular thickening on SD-0CT}

Twenty-four per cent $(n=84)$ of eyes had a degree of foveal or parafoveal macular thickening based on criteria described above (table 1). Interestingly, $70 \%$ and $67 \%$ of eyes with M1 maculopathy on FP and MC, respectively, demonstrated no macular thickening on SD-OCT.

Table 2 Intergraders and intermodality level of agreement grading M1 maculopathy as defined by DES

\begin{tabular}{ll}
\hline & Cohen's $\kappa$ coefficient \\
\hline Grading on FP: OK vs SP & $0.91(\mathrm{p}<0.0001)$ \\
Grading on MC: OK vs SP & $0.82(\mathrm{p}<0.0001)$ \\
OK grading FP vs MC & $0.76(\mathrm{p}<0.0001)$ \\
SP grading FP vs MC & $0.72(\mathrm{p}<0.0001)$ \\
Overall grading FP vs MC & $0.76(\mathrm{p}-<0.0001)$ \\
\hline
\end{tabular}

DES, Diabetic Eye Screening; FP, fundus photography; MC, multicolour.

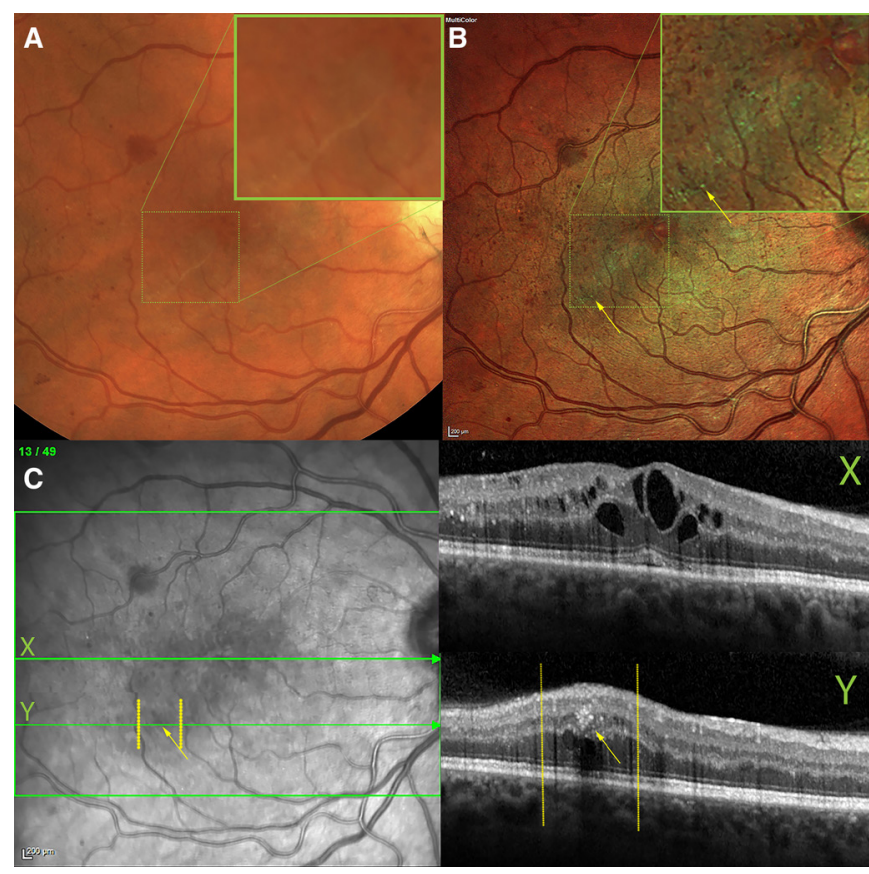

Figure 3 Macular exudate and thickening are evident on multicolour (B) and SD-OCT (C), but missing from fundus photo (A). There is a linearly shaped cluster of exudates on multicolour $(B)$ that are missed on fundus photo $(A)$, even at high magnification (inset). Two cross-sectional OCT scans are shown in $(\mathrm{C})$, with its near infrared fundus registration image on the left: $X$, centred on the fovea, shows cystoid macular oedema; $Y$, is a line that cross-sections one of the exudates, marked with the yellow arrow, seen on the multicolour image $(B)$ but not on the fundus photo $(A)$ or on the infrared reflectance channel (C). SD-OCT, spectral domain optical coherence tomography.

Among the eyes with OCT macular thickening, $56 \%$ and $50 \%$ had no M1 maculopathy on FP and MC, respectively, and $44 \%$ of times had no M1 maculopathy either on FP or on MC.

\section{CSMO based on SD-OCT}

Eighteen eyes had CSMO on OCT, characterised by spongiform or cystoid retinal thickening causing CST $\geq 370 \mu \mathrm{m}$ or thickening $>390 \mu \mathrm{m}$ of any of the ETDRS grid inner sectors (table 1). MC and FP did not detect M1 maculopathy in one $(0.6 \%)$ and five eyes $(28 \%)$, respectively.

Five patients $(1.4 \%)$ had a CST $\geq 400 \mu \mathrm{m}$, of whom only one had no M1 maculopathy on MC but four had no M1 maculopathy on FP (figure 3).

\section{Detection of exudates on SD-OCT}

M1 maculopathy was not assessed systematically using SD-OCT. However, there were 19 cases of an exudate detected on SD-OCT within the 1DD of the macula, 10 of which were also detected on MC, but not on FP. In contrast, there were only three cases where an exudate was detected both on SD-OCT and FP but not on MC. 


\section{DISCUSSION}

In this study, we demonstrated that MC reliably detects M1 maculopathy with acceptable sensitivity and specificity, with a 'substantial' interobserver agreement, when compared with the current reference standard. Moreover, when synergistically integrated into a single platform with SD-OCT in a dedicated R1M1 virtual clinic not requiring parallel diabetic retinopathy grading, $\mathrm{MC}$ performs superior to FP in detecting and monitoring M1 maculopathy as described by DES.

Overall, the sensitivity and specificity of the MC are clinically acceptable and with a negative predictive value of $94 \%$ (95\% CI $91 \%$ to $97 \%$ ) in combined peripheral and central M1 maculopathy. However, when it comes to peripheral M1 maculopathy, beyond 1DD of the fovea, where DES criteria for M1 require the presence of discrete and grouped exudates, rather than any exudates, the MC sensitivity and specificity are excellent. Furthermore, MC has a comparable level of intergrader agreement to FP. Most of disagreements between MC and FP arose secondary to a small central exudate not being detected by one of the modalities due to the exudate being singular and small. In the case of MC, it could be due to its confocal nature and its thin focusing plane missing the small exudate. However, the group of exudates at the periphery was almost always detected by both of the modalities. Nevertheless, it must be remembered that FP, which is currently the reference standard for detecting M1 maculopathy within the DES, can miss exudates as identified on SD-OCT as shown here and elsewhere. ${ }^{14}$ Therefore, the true sensitivity and specificity of MC in detecting M1 maculopathy based on exudates criteria are unknown and may be redefined by OCT.

One of the most pertinent issues that arose in this study was the fact that more than two-thirds of the patients with M1 maculopathy according to exudate criteria on FP or MC had no macular thickening on SD-OCT. Conversely, one-fifth of patients with no signs of maculopathy on non-stereoscopic FP or MC had some degree of macular thickening based on SD-OCT. Perhaps more worryingly, 5 out of 18 patients with CSMO were missed by FP, whereas only one was missed on MC. Missing CSMO undermines the utility of FP as a screening tool for detection of maculopathy.

After the landmark ETDRS, ${ }^{22}$ grid/focal laser photocoagulation had been the first-line therapy for DMO for a number of years. However, since the advent of new pharmacological therapeutics in the form of intravitreal anti-vascular endothelial growth factor (anti-VEGF), the laser photocoagulation has been largely superseded by anti-VEGF therapy as the first-line therapy for central involving DMO. ${ }^{23} 24$

In the era of anti-VEGF therapy for DMO, along with visual acuity, the central retinal thickness is one of the most important quantitative factors considered for treatment decision-making in the majority of clinical trials. ${ }^{24}$ This renders the current DES grading criteria for definition of diabetic maculopathy based on central exudates with limited utility. In fact, the National Institute for Health and Care Excellence (NICE) guidelines for the use of anti-VEGF for DMO do not take into account the presence of exudates. ${ }^{25}{ }^{26}$ Worryingly, the FP failed to identify a significant number of patients with CSMO. Worryingly, of the five individuals who met the CST criteria for treatment of DMO with anti-VEGF as per NICE guidelines, ${ }^{25}{ }^{26}$ only four were identified as having M1 maculopathy based on FP.

In our study, while $32 \%$ of the 345 eyes assessed in this study had M1 grading by FP criteria, only $1.4 \%$ met the criteria for anti-VEGF therapy as outlined by NICE guidelines. The prevalence of diabetic maculopathy is increasing in the UK. ${ }^{27}{ }^{28}$ The current DES-based classification and referral pathway for diabetic maculopathy may need refinement to adapt to the anti-VEGF era. If multimodality imaging inclusive of OCT is introduced at the point of referral from screening and applied in any subsequent monitoring scheme, patients may be spared unnecessary reviews in secondary care and be safely discharged back from the HES to the DES programme.

Although singular central exudates on their own are largely irrelevant in terms of treatment decision-making for DMO, detection of peripheral group of exudates may be important in treatment decision-making. This is the group MC had very high positive and negative predictive values for. These patients may not have central involving DMO to be eligible for anti-VEGF treatment but they can benefit from focal laser photocoagulation. ${ }^{24} 29$ The relative indication for focal laser can be considered in focally grouped microaneurysms and leaking capillaries, which may delay or prevent conversion into central involving DMO. ${ }^{24}$

A weakness of this study is that it does not provide a direct evaluation of MC imaging and FP within the DES programme. Our non-mydriatic fundus camera is a different model of the cameras used by our local DES programme. Moreover, simultaneous MC and FP grading would be required at the point of screening. Several patients monitored in the HES diabetic maculopathy virtual clinics had a considerable time lapse from the initial DES referral. The unexpected low prevalence of eyes with M1 grading in these clinics, while reflecting inadequate discharge policies, provided the strength for MC false-positive testing in our study.

In conclusion, the sensitivity and specificity of $\mathrm{MC}$ are comparable with that of $\mathrm{FP}$ in detecting $\mathrm{M} 1 \mathrm{macu}-$ lopathy, especially where peripheral group of exudates is concerned. The relevance of central exudates causing M1 maculopathy as detected by FP is largely diminished in the era of anti-VEGF therapy. Furthermore, FP can dangerously miss DMO and overburdens the secondary care with stable patients who can be safely followed up by DES. Detection of peripheral group of exudates not causing central involving DMO may still be relevant, as focal laser photocoagulation of leaking microaneurysms and capillaries may reduce the chance of central involving DMO. MC only detects peripheral group of 
exudates reliably but integrates synergistically in a single platform with SD-OCT to provide effective monitoring of M1 diabetic maculopathy. The need of FP is eliminated by MC/SD-OCT in dedicated R1M1 virtual clinics not requiring parallel diabetic retinopathy grading. Integrated MC/SD-OCT has the potential to reduce the burden on the overstretched secondary care by reducing the number of patients who do not require any treatment for their diabetic maculopathy and who can be safely followed up in the community.

Acknowledgements We would like to thank the staff of the Department of Ophthalmology, University Hospitals Coventry and Warwickshire, Coventry, UK for their help.

Contributors OK designed the study, collected and analysed the data, and wrote the manuscript. MMDF collected the data and critically appraised the manuscript. MC collected the data and critically appraised the manuscript. EC captured the images and critically appraised the manuscript. SP designed the study, collected the data and critically appraised the manuscript.

Funding The authors have not declared a specific grant for this research from any funding agency in the public, commercial or not-for-profit sectors.

Competing interests $\mathrm{MC}$ is paid advisor to Heidelberg Engineering, Zeiss, Alcon and Centervue. SP is paid advisor to Heidelberg Engineering, Zeiss, Novartis, Bayer and Allergan.

Patient and public involvement Patients and/or the public were not involved in the design, or conduct, or reporting, or dissemination plans of this research.

Patient consent for publication Not required.

Ethics approval This project was registered with the University Hospitals Coventry and Warwickshire NHS Trust Research, Development and Innovation Department. The Trust considered the work to be a departmental service evaluation, and as such, advised us that it did not require research ethics approval.

Provenance and peer review Not commissioned; externally peer reviewed.

Data availability statement Data are available upon request.

Open access This is an open access article distributed in accordance with the Creative Commons Attribution Non Commercial (CC BY-NC 4.0) license, which permits others to distribute, remix, adapt, build upon this work non-commercially, and license their derivative works on different terms, provided the original work is properly cited, appropriate credit is given, any changes made indicated, and the use is non-commercial. See: http://creativecommons.org/licenses/by-nc/4.0/.

ORCID iD

Obaid Kousha http://orcid.org/0000-0001-5747-3376

\section{REFERENCES}

1 JQ L, Welchowski T, Letow J. Retinal Dieases in Europe: Prevalence, incidence and healthcare needs: European Society of Retina Specialists - Euretina, 2017. Available: http://www.euretina.org/ downloads/EURETINA_Retinal_Diseases.pdf [Accessed 26 Dec 2017].

2 Williams R, Airey M, Baxter $\mathrm{H}$, et al. Epidemiology of diabetic retinopathy and macular oedema: a systematic review. Eye 2004;18:963-83.

3 England $\mathrm{PH}$. Nhs diabetic eye screening programme: overview of patient pathway, grading pathway, surveillance pathways and referral pathways 2017, 2017. Available: https://www.gov.uk/government/ uploads/system/uploads/attachment_data/file/648658/Diabetic Eye_Screening_pathway_overviews.pdf [Accessed 28 Nov 2017].

4 Romero-Aroca P. Targeting the pathophysiology of diabetic macular edema. Diabetes Care 2010;33:2484-5.

5 Das A, McGuire PG, Rangasamy S. Diabetic macular edema: pathophysiology and novel therapeutic targets. Ophthalmology 2015;122:1375-94.
6 Vinores SA, Gadegbeku C, Campochiaro PA, et al. Immunohistochemical localization of blood-retinal barrier breakdown in human diabetics. Am J Pathol 1989;134:231-5.

7 Murata T, Ishibashi T, Inomata H. Immunohistochemical detection of extravasated fibrinogen (fibrin) in human diabetic retina. Graefes Arch Clin Exp Ophthalmol 1992;230:428-31.

8 Yanoff M. Ocular pathology of diabetes mellitus. Am J Ophthalmol 1969;67:21-38.

9 England $\mathrm{PH}$. Nhs diabetic eye screening programme: grading definitions for referable disease, 2017. Available: https://www.gov. uk/government/uploads/system/uploads/attachment_data/file/ 582710/Grading_definitions_for_referrable_disease_2017_new_ 110117.pdf

10 England PH. Diabetic eye screening: feature based grading forms, 2012. Available: https://www.gov.uk/government/uploads/system/ uploads/attachment_data/file/402295/Feature_Based_Grading Forms V1 4 1Nov12 SSG.pdf

11 England PH. Diabetic eye screening: guidance on camera approval, 2016. Available: https://www.gov.uk/government/publications/ diabetic-eye-screening-approved-cameras-and-settings/diabeticeye-screening-guidance-on-camera-approval

12 Kernt M, Schaller UC, Stumpf C, et al. Choroidal pigmented lesions imaged by ultra-wide-field scanning laser ophthalmoscopy with two laser wavelengths (Optomap). Clin Ophthalmol 2010;4:829-36.

13 Vingolo EM, Esposito M, Librando A, et al. New retinal imaging for the visualization and analysis of vitreoretinal interface (VRI) by short-wavelength scanning laser ophthalmoscope (swSLO). Clin Ophthalmol 2011;5:1007-10.

14 Graham KW, Chakravarthy U, Hogg RE, et al. Identifying features of early and late age-related macular degeneration: a comparison of multicolor versus traditional color fundus photography. Retina 2018;38:1751-8.

15 Ben Moussa N, Georges A, Capuano V, et al. Multicolor imaging in the evaluation of geographic atrophy due to age-related macular degeneration. Br J Ophthalmol 2015;99:842-7.

16 Kang SW, Park CY, Ham D-I. The correlation between fluorescein angiographic and optical coherence tomographic features in clinically significant diabetic macular edema. Am J Ophthalmol 2004:137:313-22.

17 Koleva-Georgieva DN, Sivkova NP. Types of diabetic macular edema assessed by optical coherence tomography. Folia Med 2008;50:30-8.

18 , Wells JA, Glassman AR, et al, Diabetic Retinopathy Clinical Research Network. Aflibercept, bevacizumab, or ranibizumab for diabetic macular edema. N Engl J Med 2015;372:1193-203.

19 Panozzo G, Parolini B, Gusson E, et al. Diabetic macular edema: an OCT-based classification. Semin Ophthalmol 2004;19:13-20.

20 Grover S, Murthy RK, Brar VS, et al. Comparison of retinal thickness in normal eyes using Stratus and spectralis optical coherence tomography. Invest Ophthalmol Vis Sci 2010;51:2644-7.

21. Landis JR, Koch GG. The measurement of observer agreement for categorical data. Biometrics 1977;33:159-74

22 Photocoagulation for diabetic macular edema. early treatment diabetic retinopathy study report number 1 . early treatment diabetic retinopathy study Research Group. Arch Ophthalmol 1985;103:1796-806.

23 Distefano LN, Garcia-Arumi J, Martinez-Castillo V, et al. Combination of anti-VEGF and laser photocoagulation for diabetic macular edema: a review. J Ophthalmol 2017:2017:1-7.

24 Schmidt-Erfurth U, Garcia-Arumi J, Bandello F, et al. Guidelines for the management of diabetic macular edema by the European Society of retina specialists (EURETINA). Ophthalmologica 2017;237:185-222

25 (NICE) NIfHaCE. Aflibercept for treating diabetic macular oedema, 2015.

26 (NICE) NIfHaCE. Ranibizumab for treating diabetic macular oedema, 2013.

27 González ELM, Johansson S, Wallander M-A, et al. Trends in the prevalence and incidence of diabetes in the UK: 1996-2005. J Epidemiol Community Health 2009;63:332-6.

28 Evans JMM, Barnett KN, Ogston SA, et al. Increasing prevalence of type 2 diabetes in a Scottish population: effect of increasing incidence or decreasing mortality? Diabetologia 2007;50:729-32.

29 Diabetic retinopathy guidelines 2012 . 